\title{
FATORES ASSOCIADOS À SÍNDROME RESPIRATÓRIA AGUDA GRAVE POR COVID-19 NA POPULAÇÃO PEDIÁTRICA: ESTUDO DE CASO- CONTROLE
}

\section{Autores}

Nicholas de Albuquerque Corrêa Duarte Mayara Cristina Silva dos Santos' João Grégory da Silva Soares', Marília Rocha Kintschev', Hugo D. Hoffmann-Santos, $\mathrm{PhD}^{2}$

1. Discente do curso de medicina do Centro Universitário de Várzea Grande (UNIVAG).

2. Docente do curso de medicina do Centro Universitário de Várzea Grande (UNIVAG).

\section{RESUMO}

Introdução: COVID-19 é uma infecção causada pelo novo coronavírus (SARS-CoV-2), sendo declarada pandemia no início de 2020. É responsável por causar Síndrome Respiratória Aguda Grave (SRAG) em todas faixas etárias, inclusive pediátrica. Objetivo: Analisar fatores associados à SRAG em pacientes pediátricos infectados por SARSCoV-2 comparando aos pacientes com Influenza. Métodos: Estudo caso controle realizado com 3133 pacientes pediátricos com SRAG no Brasil entre março e setembro de 2020, através de dados do Sistema de Informação de Vigilância Epidemiológica da Gripe (SIVEP). Dividido em caso (COVID-19) e controle (Influenza), sendo analisadas variáveis sociodemográficas, sintomáticas, comorbidades e evolutivas. Resultados: Caso com 2452 pacientes e controle com 681 pacientes, evidenciado na análise bivariada associação positiva com os casos: faixa etária dos 10-17 anos, área com surto gripal, imunossupressão, diabetes, diarreia e óbito. Por outro lado, associação negativa com tosse e saturação baixa na análise multivariada. Conclusão: São fatores de risco em pacientes COVID-19 possuir idade entre 10-17 anos, morar em área com surto gripal, imunodepressão, diabetes e diarreia. Além de apresentarem maior mortalidade comparada aos pacientes com Influenza.

Palavras-Chave: COVID-19; Influenza; Síndrome Respiratória Grave Aguda; Fator de Risco; Pediatria.

\section{ABSTRACT}

Introduction: COVID-19 is an infection caused by the new coronavirus (SARS-CoV-2), being declared pandemic in early 2020. It is responsible for causing Severe Acute Respiratory Syndrome (SARS) in all age groups, including pediatric. Objective: To analyze factors associated with SARS 
1. Cui J, Li F, Shi Z-L. Origin and evolution of pathogenic coronaviruses. Nat Rev Microbiol. 2019; 17: 181-192. Disponível em: https://www. nature.com/articles/s41579-018-0118-9

2. Johns Hopkins University \& Medicine. Coronavírus Resource Center. [Update on: 2020 oct 20]. Disponível em: https:// coronavirus.jhu.edu/data in pediatric patients infected with SARS-CoV-2 compared to patients with Influenza. Methods: A case-control study carried out with 3133 pediatric patients with SARS in Brazil between March and September 2020, using data from the Brazilian Influenza Epidemiological Surveillance Information System (SIVEP). Divided into case (COVID-19) and control (Influenza), sociodemographic, symptomatic, comorbidities (and evolutionary variables were analyzed. Results: Case with 2452 patients and control with 681 patients, evidenced in the bivariate analysis positive association with the cases: age group of 10-17 years, area with flu outbreak, immunosuppression, diabetes, diarrhea and death. On the other hand, negative association with cough and low saturation in the multivariate analysis. Conclusion: Age between 1017 years, living in an area with a flu outbreak, immunosuppression, diabetes and diarrhea are risk factors associated with SARS-CoV-2 infection. In addition, these patients presented a higher mortality compared to patients with Influenza.

Keywords: COVID-19; Influenza; Severe Acute Respiratory Syndrome; Risk Factors; Pediatrics.

\section{INTRODUÇÃO}

Segundo a Organização Mundial de Saúde (OMS), doença COVID-19 é uma infecção causada pelo novo coronavírus (SARSCoV-2), pertencente à família betacoronavírus, descrita pela primeira vez em meados de 1960, responsável por infectar o homem e animais, como o morcego. ${ }^{1}$ A primeira epidemia por um vírus da mesma família ocorreu na China em 2002, provocando Síndrome Respiratória Aguda Grave (SRAG) em milhares de pessoas e, desde então, não haviam sido registrados novos casos. No final de 2019, a cidade de Wuhan na China foi o epicentro da pandemia, a qual perdura até os dias atuais. ${ }^{1}$ Segundo os relatórios epidemiológicos da Universidade Johns Hopkins, o número de casos já ultrapassa 40 milhões, com mais de 1 milhão de óbitos. ${ }^{2}$

Vários estudos em andamento tentam desvendar o mecanismo fisiopatológico, os aspectos imunológicos e a epidemiologia da 
4. Wang C, Horby PW, Hayden FG, Gao GF. A novel coronavírus outbreak of global health concern. The Lancet. 2020; 395 (10223): 470473. Disponível em: https://pubmed.ncbi.nlm. nih.gov/31986257/

5. Deville JG, Song E, Ouellette CP. Coronavirus disease 2019 (COVID-19): Clinical manifestations and diagnosis in children. Post TW, ed. UpToDate. Waltham, MA: UpToDate Inc. https://www.uptodate.com (Acessado em Outubro 25, 2020.)

6. Götzinger F, Santiago-García B, NogueraJulián $A$, et al. COVID-19 in children and adolescents in Europe: a multinational multicentre cohort study Lancet Child Adolesc Health. 2020; 4 (9): 653-661.

Disponível em: https://pubmed.ncbi.nlm.nih. gov/32593339/

7. McIntosh K. Coronavirus disease 2019 (COVID-19): Epidemiology, virology, and prevention. Uptodate, Aug. 2020. Post TW, ed. UpToDate. Waltham, MA: UpToDate Inc. https://www.uptodate.com (Acessado em 25 de outubro de 2020)

8. Kim L, Whitaker $\mathrm{M}$, O'Halloran $\mathrm{A}$, et al. Hospitalization Rates and Characteristics of Children Aged $<18$ Years Hospitalized with Laboratory-Confirmed COVID-19 - COVIDNET, 14 States, March 1-July 25, 2020. MMWR Morb Mortal Wkly Rep. 2020; 69 (24): 759-

765. Disponível em: https://pubmed.ncbi.nlm nih.gov/32555134

9. Stokes EK, Zambrano LD, Anderson KN, et al. Coronavirus Disease 2019 Case Surveillance - United States, January 22-May 30, 2020. MMWR Morb Mortal Wkly Rep. 2020; 69 (24): 759-765. Disponível em https://pubmed.ncbi.nlm.nih.gov/32555134/

10. Mehta P, McAuley DF, Brown M, et al. COVID-19: consider cytokine storm syndromes and immunosuppression. The Lancet. 2020; 395 (10229): 1033-1034.

Disponível em: https://pubmed.ncbi.nlm.nih. gov/32192578/

11. Yonker LM, Shen K, Kinane TB. Lessons unfolding from pediatric cases of COVID-19 disease caused by SARS-CoV-2 infection. Pediatr Pulmonol. 2020; 55 (5): 1085-1086 Disponível em: https://pubmed.ncbi.nlm.nih. gov/32243722/
COVID-19. Porém, a maioria destes avaliou a população adulta, por isso, ainda é um desafio a compreensão dessa patologia na população pediátrica. Crianças de todas as idades podem contrair a infecção, mas parecem ser menos afetadas sintomaticamente do que os adultos. ${ }^{4}$ Segundo uma coorte multicêntrica de 582 crianças europeias com SRAG causada pelo SARS-CoV-2, a faixa etária mais acometida está entre 10-18 anos. $^{5}$

O modo de transmissão é, principalmente, através de gotículas contaminadas presentes na tosse ou espirro, mas, a presença do vírus em superfície de contato também foi relatada. A maioria dos casos em crianças resulta da exposição domiciliar, geralmente, com um adulto como o caso índice, e também relatos da exposição por professores ou funcionários da escola. O papel das crianças na transmissão para outras pessoas ainda não é claro, no entanto, crianças mais velhas e adolescentes parecem ser capazes de transmitir com eficácia. ${ }^{6}$

Vários fatores de risco são implicados para a população pediátrica, desde fatores socioeconômicos (pobreza, acesso limitado a serviços de saúde famílias multigeracionais), étnicos (negros, latinos e hispânicos) e até mórbidos como doenças cardíacas congênitas ou adquiridas, doença renal crônica, imunodepressão e diabetes. ${ }^{5,7}$ Dentre as faixas etárias, a taxa cumulativa de hospitalizações é maior em lactentes. ${ }^{8}$

O fato é que a infecção apresenta um amplo espectro clínico, indo de sintomas semelhantes ao resfriado comum até franca insuficiência respiratória. Nos lactentes, predomina a recusa alimentar e febre de origem obscura. Em escolares e adolescente, febre, tosse, cefaleia e odinofagia prevaleceram. ${ }^{9}$ Em uma revisão sistemática com 7.480 crianças com a infecção, apenas $2 \%$ eram graves (dispneia, cianose, hipoxemia) e 0,7\% eram críticos (SARS, insuficiência respiratória aguda, choque). ${ }^{10,11}$

O motivo pelo qual a COVID-19 é menos comum e mais branda em crianças ainda não está claro, uma possibilidade é que as crianças tenham uma resposta imunológica menos intensa ao vírus do que os adultos. $^{12}$

O objetivo deste estudo foi analisar os fatores associados à SRAG em pacientes pediátricos com COVID-19 comparando aos pacientes acometidos pelo vírus Influenza no Brasil. 
12. Zachariah $\mathrm{P}$, Johnson $\mathrm{CL}$, Halabi $\mathrm{KC}$, et al. Epidemiology, Clinical Features, and Disease Severity in Patients With Coronavirus Disease 2019 (COVID-19) in a Children's Hospital in New York City, New York. JAMA Pediatr. 2020; 174 (10): e202430. Disponível em: https:// pubmed.ncbi.nlm.nih.gov/32492092/

\section{MÉTODOS}

Trata-se de estudo do tipo caso-controle realizado com pacientes pediátricos acometidos por SRAG no Brasil entre março e setembro de 2020. O banco de dados utilizado para a pesquisa foi obtido através das Notificações de Síndrome Respiratória Aguda Grave que é usado em todo território nacional, vinculadas ao Sistema de Informação de Vigilância Epidemiológica da Gripe (SIVEP-Gripe). Os critérios de inclusão foram SRAG, nacionalidade brasileira e idade entre 0 a 17 anos, sendo o caso (COVID-19) e o controle (Influenza) definidos por RT-PCR (sigla em inglês para Reverse-Transcriptase Polymerase Chain Reaction, ou reação da transcriptase reversa seguida pela reação em cadeia da polimerase).

A análise bivariada foi realizada para avaliar os fatores associados à COVID-19 na população pediátrica e se utilizou do teste qui-quadrado de Mantel-Haenszel e da odds ratio como medida de associação e este teste foi realizado no software Epi Info 7.2 (CDC, Atlanta,EUA).A análise multivariada foi realizada por meio da regressão logística binária, incluindo no modelo as variáveis com significância estatística da análise bivariada, sendo o ajuste do modelo avaliado pelo Goodness-of-fit test de Hosmer e Lemeshow, estas análises foram realizadas por meio do software Stata 13.0 (College Station, Texas, EUA). Foi considerado significância estatística p-valor < 0,05.

\section{RESULTADOS}

A amostra foi composta por 3.133 pacientes pediátricos, sendo a maior parte composta de indivíduos de 0 a 9 anos, sexo masculino, etnia branca/parda, residentes na região Sudeste, em zona urbana/ periurbana, não proveniente de área com surto gripal e com diagnóstico de COVID-19 por RT-PCR (tabela 1). 
Tabela 1. Avaliação descritiva da amostra na população pediátrica com SRAG por COVID-19 e Influenza no Brasil entre março e setembro de 2020.

\begin{tabular}{|c|c|c|c|c|c|}
\hline Variáveis & $\mathbf{N}$ & $\%$ & Variáveis & $\mathbf{N}$ & $\%$ \\
\hline Idade & & & Zona & & \\
\hline 0 a 9 anos & 2260 & 72,14 & Urbana/Periurbana & 2630 & 83,95 \\
\hline 10 a 17 anos & 873 & 27,86 & Rural & 207 & 6,61 \\
\hline Sexo & & & Ignorado & 296 & 9,45 \\
\hline Masculino & 1679 & 53,61 & Área com surto gripal & & \\
\hline Feminino & 1453 & 46,39 & $\operatorname{Sim}$ & 500 & 15,96 \\
\hline Etnia & & & Não & 1912 & 61,03 \\
\hline Branco/ Pardo & 2114 & 93,79 & Ignorado & 721 & 23,01 \\
\hline Outros & 140 & 6,21 & Gestante & & \\
\hline Região & & & Sim & 49 & 1,56 \\
\hline Norte & 502 & 16,02 & Não & 315 & 10,05 \\
\hline Nordeste & 977 & 31,18 & Ignorado & 2769 & 88,38 \\
\hline Centro-oeste & 282 & 9,00 & Diagnóstico final & & \\
\hline Sudeste & 1259 & 40,19 & COVID-19 & 2452 & 78,26 \\
\hline Sul & 113 & 3,61 & SRAG por Influenza & 681 & 21,74 \\
\hline
\end{tabular}

As seguintes variáveis apresentaram associação positiva com COVID-19 na análise bivariada: idade entre 10 e 17 anos, indivíduo proveniente de área com surto gripal, diarreia, imunodepressão, diabetes e óbito. Todavia, no mesmo tipo de análise, apresentaram associação negativa: presença de tosse, febre, dispneia, dor de garganta, desconforto respiratório, SpO2 <95\%, vacinação para gripe, asma, hospitalização e tratamento antiviral (tabela 2).

Tabela 2. Fatores associados à SRAG por COVID-19 na população pediátrica em comparação aos pacientes com Influenza da amostra em país no Brasil entre março e setembro de 2020. $(\mathbf{N}=3133)$

\begin{tabular}{|c|c|c|c|c|}
\hline \multirow[t]{2}{*}{ Variáveis } & COVID-19 & Influenza & \multirow[t]{2}{*}{ OR (IC95\%) } & \multirow[t]{2}{*}{ p-valor } \\
\hline & $\mathrm{N}=\mathbf{2 4 5 2}$ & $N=681$ & & \\
\hline \multicolumn{5}{|l|}{ 1. Sociodemográficas } \\
\hline \multicolumn{5}{|l|}{ Idade } \\
\hline 10 a 17 anos & $757(30,87)$ & $116(17,03)$ & $2,17(1,75-2,70)$ & $<0,001$ \\
\hline 0 a 9 anos & $1695(69,13)$ & $565(82,97)$ & 1,00 & \\
\hline \multicolumn{5}{|l|}{ Sexo } \\
\hline Masculino & $1303(53,16)$ & $376(55,21)$ & $0,92(0,77-1,09)$ & 0,342 \\
\hline Feminino & $1148(46,84)$ & $305(44,79)$ & 1,00 & \\
\hline \multicolumn{5}{|l|}{ Etnia } \\
\hline Branco/Pardo & $1634(93,64)$ & $480(94,30)$ & $0,88(0,58-1,35)$ & 0,585 \\
\hline Outras & $111(6,36)$ & $29(5,70)$ & 1,00 & \\
\hline \multicolumn{5}{|l|}{ Zona } \\
\hline Urbana/Periurbana & $2033(92,28)$ & $597(94,16)$ & $0,74(0,51-1,06)$ & 0,108 \\
\hline Rural & $170(7,72)$ & $37(5,84)$ & 1,00 & \\
\hline \multicolumn{5}{|l|}{ Área com surto gripal } \\
\hline $\operatorname{Sim}$ & $440(23,98)$ & $60(10,40)$ & $2,71(2,03-3,62)$ & $<0,001$ \\
\hline Não & $1395(76,02)$ & $517(89,60)$ & 1,00 & \\
\hline
\end{tabular}




\begin{tabular}{|c|c|c|c|c|}
\hline \multicolumn{5}{|c|}{ 2. Sintomatologia } \\
\hline \multicolumn{5}{|c|}{ Tosse } \\
\hline Sim & $1449(67,27)$ & $616(91,94)$ & $0,18(0,13-0,24)$ & $<0,001$ \\
\hline Não & $705(32,73)$ & $54(8,06)$ & 1,00 & \\
\hline \multicolumn{5}{|l|}{ Febre } \\
\hline Sim & $1725(76,63)$ & $654(96,60)$ & $0,11(0,07-0,17)$ & $<0,001$ \\
\hline Não & $526(23,37)$ & $23(3,40)$ & 1,00 & \\
\hline \multicolumn{5}{|l|}{ Dispneia } \\
\hline Sim & $1181(56,78)$ & $438(66,57)$ & $0,65(0,54-0,79)$ & $<0,001$ \\
\hline Não & $899(43,22)$ & $220(33,43)$ & 1,00 & \\
\hline \multicolumn{5}{|l|}{ Diarreia } \\
\hline Sim & $347(18,27)$ & $83(13,76)$ & $1,40(1,08-1,81)$ & 0,010 \\
\hline Não & $1552(81,73)$ & $520(86,24)$ & 1,00 & \\
\hline \multicolumn{5}{|c|}{ Dor de garganta } \\
\hline Sim & $408(22,16)$ & $186(30,95)$ & $0,63(0,51-0,77)$ & $<0,001$ \\
\hline Não & $1433(77,84)$ & $415(69,05)$ & 1,00 & \\
\hline \multicolumn{5}{|c|}{ Desconforto respiratório } \\
\hline Sim & $1167(56,79)$ & $526(80,18)$ & $0,32(0,26-0,40)$ & $<0,001$ \\
\hline Não & $888(43,21)$ & $130(19,82)$ & 1,00 & \\
\hline \multicolumn{5}{|c|}{ Saturação < 95\% } \\
\hline Sim & $797(40,68)$ & $325(51,42)$ & $0,64(0,54-0,77)$ & $<0,001$ \\
\hline Não & $1162(59,32)$ & $307(48,58)$ & 1,00 & \\
\hline \multicolumn{5}{|l|}{ Vacinado } \\
\hline Sim & $163(18,67)$ & $110(30,14)$ & $0,53(0,40-0,70)$ & $<0,001$ \\
\hline Não & $710(81,33)$ & $255(69,86)$ & 1,00 & \\
\hline
\end{tabular}

\section{Comorbidades}

\section{Pneumopatia}

Sim

Não

Cardiopatia

$$
\text { Sim }
$$

Não

Asma

$$
\text { Sim }
$$

Imunodepressão

$$
\text { Sim }
$$

Não

Diabetes

Sim $49(8,58)$

Não

Hematológicas

$$
\text { Sim }
$$

Não

Neurológica

$$
\text { Sim }
$$

Não

Renal

morbidades
Sim
Não
Sitia
Não
Sim
Não
depressão
Sim
Não
Sim
Não
Simógicas
Não
lógica
Sim
Não
Sim

$$
\begin{gathered}
52(9,39) \\
502(90,61)
\end{gathered}
$$

$92(15,67)$

$495(84,33)$

$134(23,02)$

$448(76,98)$

$136(22,55)$

$467(77,45)$

$522(91,42)$

$52(9,27)$
$509(90,73)$

$141(24,31)$

$439(75,69)$
$18(11,61)$

$137(88,39)$

$16(10,46)$

$137(89,54)$

$61(37,65)$

$101(62,35)$

$17(10,97)$

$138(89,03)$

$1(0,67)$

$149(99,33)$

$$
\begin{gathered}
7(4,58) \\
146(95,42)
\end{gathered}
$$

$37(23,87)$

$118(76,13)$

$$
\begin{gathered}
0,78(0,44-1,39) \\
1,00
\end{gathered}
$$

0,411

$$
\begin{gathered}
1,59(0,90-2,79) \\
1,00
\end{gathered}
$$$$
0,103
$$

$$
\begin{array}{cl}
0,49(0,34-0,71) & <0,001 \\
1,00 &
\end{array}
$$

$$
\begin{gathered}
2,36(1,37-4,05) \\
1,00
\end{gathered}
$$

$$
\begin{gathered}
13,98(1,91-102,1) \quad<0,001 \\
1,00
\end{gathered}
$$

$$
\begin{gathered}
2,13(0,94-4,79) \quad 0,061 \\
1,00
\end{gathered}
$$

$1,02(0,67-1,55) \quad 0,909$

1,00

$4(2,74)$

$2,27(0,79-6,51)$

0,117

Não

$33(6,01)$ $142(97,26)$ 1,00 


\begin{tabular}{lcccc}
\hline 4. Evolução & & & & \\
\hline Hospitalização & $2086(89,34)$ & $657(97,33)$ & $0,22(0,14-0,37)$ & $<0,001$ \\
Sim & $249(10,66)$ & $18(2,67)$ & 1,00 & \\
Não & & & & \\
Terapia Intensiva & $612(31,53)$ & $200(32,41)$ & $0,96(0,79-1,16)$ & 0,680 \\
Sim & $1329(68,47)$ & $417(67,59)$ & 1,00 & \\
Não & $818(44,41)$ & $266(47,42)$ & $0,88(0,73-1,07)$ & 0,21 \\
Suporte ventilatório & $1024(55,59)$ & $295(55,58)$ & 1,00 & \\
Sim & & & & \\
Não & $563(27,94)$ & $448(71,91)$ & $0,15(0,12-0,18)$ & $<0,001$ \\
Antiviral & $1452(72,06)$ & $175(28,09)$ & 1,00 & \\
Sim & & & & \\
Não & $276(16,53)$ & $31(5,24)$ & $3,58(2,44-5,26)$ & $<0,001$ \\
Desfecho & $1394(83,47)$ & $561(94,76)$ & 1,00 & \\
Óbito & &
\end{tabular}

Após análise multivariada, observou-se que pacientes pediátricos com COVID-19 receberam tratamento com antivirais 67\%, tiveram chance 55\% menor de SpO2 < 95\% e 78\% menor chance de manifestar tosse como sintoma comparado aos pacientes pediátricos com SRAG por influenza (tabela 3).

Tabela 3. Análise multivariada dos fatores independentes e associados à COVID-19 na população pediátrica quando comparados com casos confirmados de SRAG por Influenza entre março e setembro de 2020.

\begin{tabular}{|c|c|c|}
\hline Variáveis & OR (IC95\%) & P-valor \\
\hline \multicolumn{3}{|l|}{ Antiviral } \\
\hline Sim & $0,33(0,16-0,68)$ & 0,003 \\
\hline Não & 1,00 & \\
\hline \multicolumn{3}{|l|}{$\mathrm{SpO}_{2}$} \\
\hline$<95 \%[$ baixa, risco] & $0,45(0,20-0,98)$ & 0,046 \\
\hline$\geq 95 \%[$ alta, não risco] & 1,00 & \\
\hline \multicolumn{3}{|l|}{ Tosse } \\
\hline Presente & $0,22(0,07-0,72)$ & 0,012 \\
\hline Ausente & 1,00 & \\
\hline
\end{tabular}

Modelo ajustado por: sexo, asma, idade, desconforto respiratório, febre, garganta, hospital, imunodepressão, vacina e vômito. Goodness-of-fit test $(\mathrm{p}=0,14)$. 
13. Coronavirus Disease 2019 in Children - Estados Unidos, 12 de fevereiro a 2 abril de 2020. MMWR Morb Wkly Rep 2020; 69 (14): 422-426. Disponível em: http://dx.doi. org/10.15585/mmwr.mm6914e4

14. Dong $Y$, Mo X, Hu Y, Qi X, Jiang F, Jiang $Z$, et al. Epidemiology of COVID-19 among children in China. Pediatrics. 2020; 145 (6): e20200702. Disponível em: https://pubmed. ncbi.nlm.nih.gov/32179660/

15. Ouldali N, Yang DD, Madhi F, et al. Factors Associated With Severe SARS CoV-2 Infection. Pediatrics. 2021; 147 (3): e2020023432. Disponível em: https://doi org/10.1542/peds.2020-023432

16. Phucharoen $\mathrm{C}$, et al. The characteristics of COVID-19 transmission from case to

high-risk contact, a statistical analysis from contact tracing data. EClinicalMedicine. 2020; 27: 100543. Disponível em: https://doi. org/10.1016/j.eclinm.2020.100543

17. Sociedade Brasileira de Pediatria. Síndrome de Cushing e COVID-19 em Pediatria. Rio de Janeiro: SBP, 2020. Disponível em: https://www.sbp.com.br/

\section{DISCUSSÃO}

Em nossos dados, houve maior prevalência de COVID-19 foi observada em indivíduos do sexo masculino. A maior ocorrência neste grupo de indivíduos é concordante com prevalência nos Estados Unidos, onde se constatou que dos 2.572 casos de COVID-19 em crianças, 57\% eram do sexo masculino. A faixa etária de 0-9 anos foi mais acometida, achado semelhante à média de idade de 7 anos encontrada em análise retrospectiva com 2135 pacientes, segundo Dong (2020). Por outro lado, em nosso estudo a faixa etária de 10-17 anos foi associada a um maior risco de SRAG por COVID-19, condizente com Ouldali (2020), no qual um dos fatores independentemente associados à gravidade foram idade $\geqslant 10$ anos (OR= 3,4; IC95\%: 1,1-10,3). Crianças provenientes de áreas com surto gripal foram mais infectadas, condizente com a alta transmissibilidade do Sars-Cov-2.13,14,15,16

Os sintomas mais comuns foram febre e tosse, se correlacionando com os achados de Dong (2020), no qual tosse e febre corresponderam a $48.5 \%$ e $41.5 \%$ dos sintomas, respectivamente. Segundo Deville (2020), as crianças tendem a apresentar sintomas mais brandos, o que corrobora nosso achado: menor associação da infecção pelo Sars-Cov-2 com dispneia, desconforto respiratório e saturação menor que $95 \%$ em pacientes pediátricos. A diarreia foi observada como sintoma menos encontrado, aproximadamente $18 \%$ dos pacientes com COVID-19 neste estudo e em 9\% dos pacientes, segundo Dong (2020). Porém, foi o único sintoma que apresentou associação com SRAG por COVID-19 em comparação com o grupo controle. Ademais, Deville (2020) demonstra que dentre os sintomas gastrointestinais, os mais relatados são diarreia, dor abdominal e vômitos, podendo surgir sem sintomas respiratórios..$^{13,14}$

Uma porcentagem de $22,55 \%$ de um total de 467 pacientes com diagnóstico de COVID-19 apresentou imunossupressão, além da condição ter sido associada ao SARS-CoV-2 quando analisada em tabela bivariada. Apesar da imunossupressão não ter sido especificada, é reconhecido que pacientes com sistema imune enfraquecido possuem risco aumentado para infecção pelo SARS-CoV-2 e suas complicações, principalmente pacientes em uso de corticoides. ${ }^{17}$ Além disso, a Rede de Imunodeficiência Primária do Reino Unido elenca outras imunodeficiências que possuem risco extremamente 
13. Coronavirus Disease 2019 in Children - Estados Unidos, 12 de fevereiro a 2 abril de 2020. MMWR Morb Wkly Rep 2020; 69 (14): 422-426. Disponível em: http://dx.doi. org/10.15585/mmwr.mm6914e4

18. Sociedade Paranaense de Pediatria. Doenças pediátricas e formas graves de covid-19. Curitiba: SPP, 2020. Disponível em: http://www.spp.org.br/

19. Bailey LC, et al. Pediatric Patients Tested for Severe Acute Respiratory Syndrome

Coronavirus 2 Across the United States. JAMA

Pediatr. 2021; 175 (2): 176-184. Disponível em: doi: 10.1001/jamapediatrics.2020.5052.

20. Sociedade Brasileira de Pediatria. Síndrome Inflamatória Multissistêmica em Crianças e Adolescentes provavelmente associada à COVID-19: Uma apresentação aguda, grave e potencialmente fatal. Rio de Janeiro: SBP, 2020. Disponível em: https:// www.sbp.com.br/

21. Abrams EM, et al. Pediatric asthma and COVID-19: The known, the unknown, and the controversial. Pediatric Pulmonology. 2020; 55: 3573-3578. Disponível em: https://doi. org/10.1002/ppul.25117

22. Zein J., Whelan G, Erzurum S. Safety of Influenza Vaccine during COVID-19. Journa of Clinical and Translational Science. 2020; 1-6. Disponível em: https://doi.org/10.1017/ cts. 2020.543 alto para o desenvolvimento de COVID-19 e suas complicações, como pacientes com imunodeficiências de células B ou T, pacientes em uso de profilaxia antibiótica ou em uso imunoglobulina. ${ }^{18}$

A análise bivariada fora observada forte associação do Diabetes Mellitus (DM) com a doença causada pelo SARS-CoV-2. Na coorte retrospectiva de Bailey (2021), os distúrbios endocrinológicos foram considerados fator de risco para COVID-19 (OR: 1,52; IC95\%:1,311,75). Porém, na população adulta é comprovadamente um fator de risco relacionado a piores desfechos. ${ }^{19,20}$

A prevalência de asma em nosso estudo foi de 23,02\% nos pacientes com SARS-CoV-2. Em análise realizada pelo Centers for Disease Control and Prevention (2020), 11,6\% dos pacientes com COVID-19 de um total de 345 casos eram portadores de doenças respiratórias crônicas. No entanto, o diagnóstico prévio de asma não se mostrou fator associado ao COVID-19 quando colocados em comparação com os pacientes com Influenza, como mostra a tabela 2. Assim, como demonstrado em outros estudos, a relação entre asma e COVID-19 não foi concretizada, necessitando de mais estudos. ${ }^{13,21}$

Uma parcela de 18\% dos indivíduos com COVID-19 recebeu vacinação contra Influenza. Segundo Zein (2020), foram comparados 4.138 indivíduos que haviam realizado vacinação com 9.082 que nunca haviam recebido vacina contra Influenza. Como resultado, não houve alteração da incidência de COVID-19 nos pacientes que receberam a vacinação e nem acréscimo de complicações (hospitalizações, por exemplo). ${ }^{22}$

O fato do número e necessidade de internação dos pacientes com SRAG por COVID-19 ter sido menor quando comparado aos pacientes com Influenza é concordante com os dados da literatura. Segundo CDC (2020), entre mais de 69.700 casos de COVID-19 em crianças, a taxa de hospitalização variou entre 2,5 a 4,1\%. Em nosso estudo, o risco de morte foi 3,58 maior nestes pacientes quando comparados com SRAG por Influenza. Tal afirmação pode estar relacionada à presença da Síndrome Inflamatória Multissistêmica em pacientes pediátricos com COVID-19, condição aguda e potencialmente fatal. ${ }^{13,20}$

Este estudo esbarrou no preenchimento irregular das fichas de notificação, o que dificultou a interpretação de alguns fatores, principalmente dados relacionados às comorbidades. No entanto, 
não encontramos na literatura nacional, até o presente momento, outro estudo buscando fatores associados a SRAG em pacientes pediátricos. Portanto, nosso estudo agregará em novas pesquisas.

\section{CONCLUSÃO}

Pacientes acima de 10 anos apresentam maior risco de SRAG por COVID-19 em comparação aos pacientes com Influenza. Ser proveniente de áreas com surtos gripais também foi considerado um fator de risco relevante. Dentre as comorbidades associadas aos casos, podemos citar a imunodepressão e a diabetes. $O$ sintoma com maior associação a SRAG causada pelo COVID-19 foi a diarreia, enquanto tosse e saturação baixa obtiveram associação negativa. Além disso, os pacientes com COVID-19 necessitaram de menos internações, usaram menos antiviral e eram menos vacinados comparados aos pacientes com Influenza. No entanto, o risco de óbito por SRAG foi maior em pacientes com COVID-19 quando comparado aos pacientes com Influenza.

\section{REFERÊNCIAS}

1. Cui J, Li F, Shi Z-L. Origin and evolution of pathogenic coronaviruses. Nat Rev Microbiol. 2019; 17: 181-192. Disponível em: https://www.nature.com/articles/s41579-018-0118-9

2. Johns Hopkins University \& Medicine. Coronavírus Resource Center. [Update on: 2020 oct 20]. Disponível em: https://coronavirus.jhu.edu/data

3. Huang C, Wang Y, Li X, et al. Clinical features of patients infected with 2019 novel coronavirus in Wuhan, China. The Lancet. 2020; 395 (10223): 497-506. Disponível em: https://pubmed.ncbi.nlm. nih.gov/31986264/

4. Wang C, Horby PW, Hayden FG, Gao GF. A novel coronavírus outbreak of global health concern. The Lancet. 2020; 395 (10223): 470-473. Disponível em: https://pubmed.ncbi.nlm.nih. gov/31986257/

5. Deville JG, Song E, Ouellette CP. Coronavirus disease 2019 (COVID-19): Clinical manifestations and diagnosis in children. Post TW, ed. UpToDate. Waltham, MA: UpToDate Inc. https://www.uptodate.com (Acessado em Outubro 25, 2020.)

6. Götzinger F, Santiago-García B, Noguera-Julián A, et al. COVID-19 in children and adolescents in 
Europe: a multinational, multicentre cohort study. Lancet Child Adolesc Health. 2020; 4 (9): 653661. Disponível em: https://pubmed.ncbi.nlm.nih.gov/32593339/

7. McIntosh K. Coronavirus disease 2019 (COVID-19): Epidemiology, virology, and prevention. Uptodate, Aug. 2020. Post TW, ed. UpToDate. Waltham, MA: UpToDate Inc. https://www.uptodate.com (Acessado em 25 de outubro de 2020)

8. Kim L, Whitaker M, O'Halloran A, et al. Hospitalization Rates and Characteristics of Children Aged $<18$ Years Hospitalized with Laboratory-Confirmed COVID-19 - COVID-NET, 14 States, March 1-July 25, 2020. MMWR Morb Mortal Wkly Rep. 2020; 69 (24): 759-765. Disponível em: https://pubmed. ncbi.nlm.nih.gov/32555134/

9. Stokes EK, Zambrano LD, Anderson KN, et al. Coronavirus Disease 2019 Case Surveillance - United States, January 22-May 30, 2020. MMWR Morb Mortal Wkly Rep. 2020; 69 (24): 759-765. Disponível em: https://pubmed.ncbi.nlm.nih.gov/32555134/

10. Mehta P, McAuley DF, Brown M, et al. COVID-19: consider cytokine storm syndromes and immunosuppression. The Lancet. 2020; 395 (10229): 1033-1034.. Disponível em: https://pubmed.ncbi. nlm.nih.gov/32192578/

11. Yonker LM, Shen K, Kinane TB. Lessons unfolding from pediatric cases of COVID-19 disease caused by SARS-CoV-2 infection. Pediatr Pulmonol. 2020; 55 (5): 1085-1086. Disponível em: https:// pubmed.ncbi.nlm.nih.gov/32243722/

12. Zachariah P, Johnson CL, Halabi KC, et al. Epidemiology, Clinical Features, and Disease Severity in Patients With Coronavirus Disease 2019 (COVID-19) in a Children's Hospital in New York City, New York. JAMA Pediatr. 2020; 174 (10): e202430. Disponível em: https://pubmed.ncbi.nlm.nih. gov/32492092/

13. Coronavirus Disease 2019 in Children - Estados Unidos, 12 de fevereiro a 2 abril de 2020. MMWR Morb Wkly Rep 2020; 69 (14): 422-426. Disponível em: http://dx.doi.org/10.15585/mmwr. $\mathrm{mm} 6914 \mathrm{e} 4$

14. Dong Y, Mo X, Hu Y, Qi X, Jiang F, Jiang Z, et al. Epidemiology of COVID-19 among children in China. Pediatrics. 2020; 145 (6): e20200702. Disponível em: https://pubmed.ncbi.nlm.nih. gov/32179660/ 
15. Ouldali N, Yang DD, Madhi F, et al. Factors Associated With Severe SARS-CoV-2 Infection. Pediatrics. 2021; 147 (3): e2020023432. Disponível em: https://doi.org/10.1542/peds.2020-023432

16. Phucharoen C, et al. The characteristics of COVID-19 transmission from case to high-risk contact, a statistical analysis from contact tracing data. EClinicalMedicine. 2020; 27: 100543. Disponível em: https://doi.org/10.1016/j.eclinm.2020.100543

17. Sociedade Brasileira de Pediatria. Síndrome de Cushing e COVID-19 em Pediatria. Rio de Janeiro: SBP, 2020. Disponível em: https://www.sbp.com.br/

18. Sociedade Paranaense de Pediatria. Doenças pediátricas e formas graves de covid-19. Curitiba: SPP, 2020. Disponível em: http://www.spp.org.br/

19. Bailey LC, et al. Pediatric Patients Tested for Severe Acute Respiratory Syndrome Coronavirus 2 Across the United States. JAMA Pediatr. 2021; 175 (2): 176-184. Disponível em: doi: 10.1001/jamapediatrics.2020.5052.

20. Sociedade Brasileira de Pediatria. Síndrome Inflamatória Multissistêmica em Crianças e Adolescentes provavelmente associada à COVID-19: Uma apresentação aguda, grave e potencialmente fatal. Rio de Janeiro: SBP, 2020. Disponível em: https://www.sbp.com.br/

21. Abrams EM, et al. Pediatric asthma and COVID-19: The known, the unknown, and the controversial. Pediatric Pulmonology. 2020; 55: 3573-3578. Disponível em: https://doi.org/10.1002/ ppul.25117

22. Zein J, Whelan G, Erzurum S. Safety of Influenza Vaccine during COVID-19. Journal of Clinical and Translational Science. 2020; 1-6. Disponível em: https://doi.org/10.1017/cts.2020.543 